\title{
Peri-operative Management and the Role of Minimally Invasive Spine Surgery in a Case of Hemophilia B
}

\author{
VNR Praveen Goparaju, Amit Chugh, Ameya Rangnekar, Vishal Kundnani \\ Department of Orthopaedics, Bombay Hospital \& Medical Research Centre, Mumbai, India
}

Received: October 12, 2021

Revised: January 5, 2022

Accepted: January 23, 2022

Corresponding Author:

VNR Praveen Goparaju, MS

Fellow - Association of Spine

Surgeons in India, Department of

Orthopaedics, Bombay Hospital \&

Medical Research Centre, 12 New

Marine Lines, Mumbai 400020,

India

Tel: +91-9160651062

Fax: +91-22-22080871

E-mail: praveen.gvnr@gmail.com
Hemophilia A and B are rare X-chromosome-linked recessive bleeding disorders caused by mutations in the genes causing abnormalities of blood clotting factors VIII and IX, respectively. Surgery in these patients will require additional planning and interaction among the surgeon, anesthetist, and a hematologist because they inevitably result in bleeding, excessive blood loss, and other life-threatening complications. The authors present a case 62-year-old male with haemophilia B and progressive neurological claudication. On plain radiographs and MRI the patient had grade 1 spondylolisthesis with lumbar canal stenosis at L4-L5 with a VAS score of 8 and ODI score of 45 and was operated with MIS-TLIF with $22 \mathrm{~mm}$ diameter tubular retractor (METRx, Medtronics) and an operating microscope. Pre-operatively, the hematologist opinion was taken and the patient was optimised by maintaining the plasma factor peak level activity according to the WFH guidelines. The patient had uneventful peri-operative period. The total hospital stay is 16 days and a VAS score of 3 and ODI score of 12 after one-year follow-up and without any notable complications. Minimally invasive surgical techniques are a better option in hemophilia patients as these techniques provide the surgeon with an excellent magnification of the operative field, which enables the use of a smaller incision, better hemostasis, and facilitates less traumatic procedures.

Key Words: Hemophilia B, Factor IX, Minimally invasive surgery, spinal fusion, Spine, Surgical blood loss

\section{INTRODUCTION}

Spine surgery in the hemophilia patient is not a well documented entity in the literature. As per the author's knowledge, there is no literature related to minimally invasive spine surgery in hemophilia. We report our experience with a patient of hemophilia B treated with minimally invasive transforaminal lumbar interbody fusion (MIS-TLIF).

\section{CASE REPORT}

After taking patient consent for purpose of the study with due care to maintain his privacy, the authors present a known case of 62-year-old male with haemophilia B and progressive neurological claudication. On plain radiographs, the patient had grade 1 spondylolisthesis according to mayerdings classification at L4-L5 with a visual analogue scale (VAS) score of 8 and Oswestry disability index (ODI) score of 45 and no symptomatic improvement with conservative management. On MRI, the patient had L4-L5 lumbar canal stenosis and the patient was operated on with MIS-TLIF (Figure 1). Pre-operatively, the hematologist opinion was taken and the patient was optimised by maintaining the plasma factor peak level activity at 60-80 IU/DL according to the world federation of hemophilia (WFH)

Copyright (C) 2022 Korean Minimally Invasive Spine Surgery Society

This is an Open Access article distributed under the terms of the Creative Commons Attribution Non-Commercial License (http://creativecommons.org/licenses/by-nc/4.0/) which permits unrestricted non-commercial use, distribution, and reproduction in any medium, provided the original work is properly cited. 

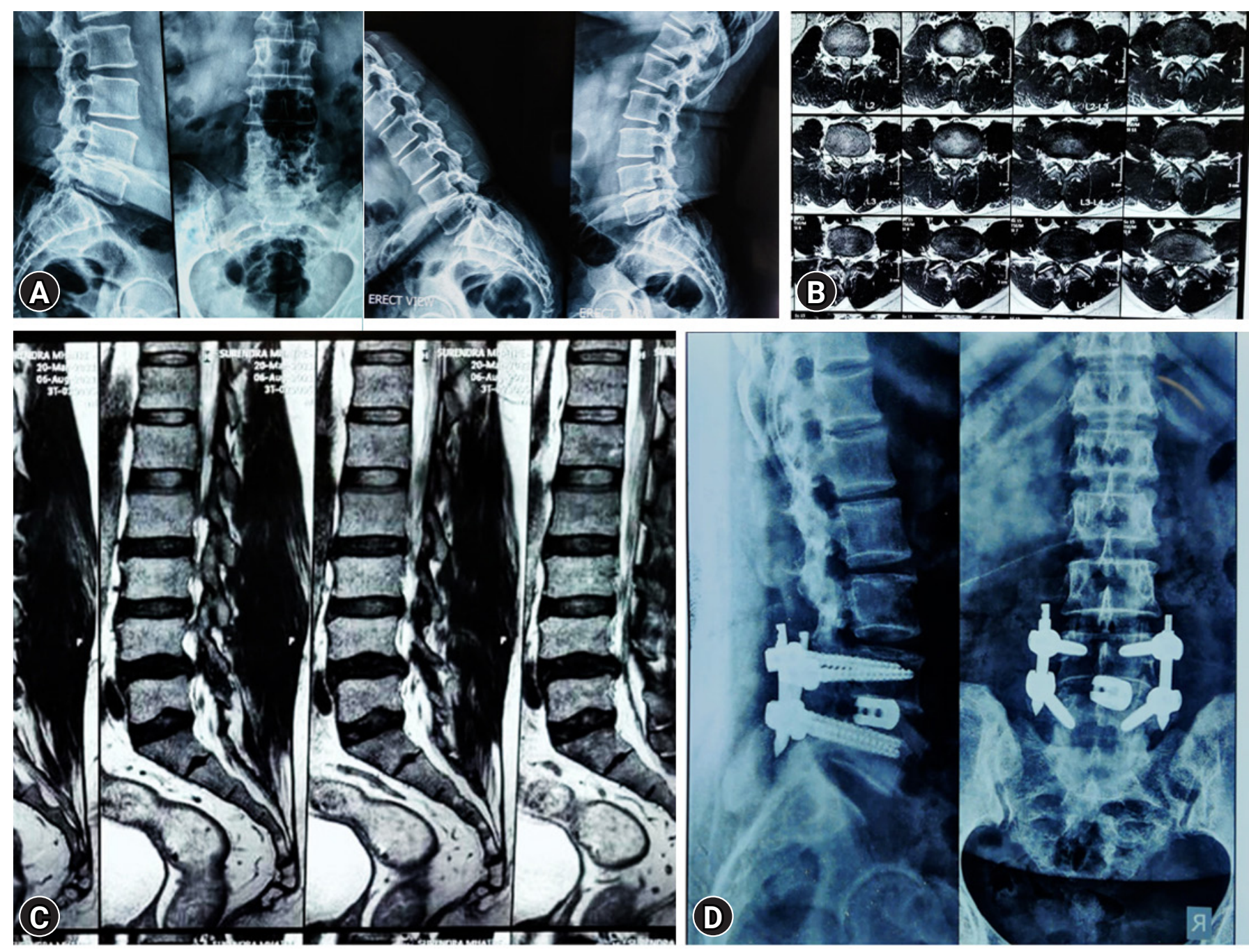

Figure 1. Index case. (A) Pre-operative plain radiographs. (B) Axial MR images. (C) Sagittal MR images. (D) Post-operative plain radiographs.

guidelines [1] after checking with activated partial thromboplastin time (APTT), and All factor allogeneic antibodies (inhibitors) levels (Table 1). Intra-operatively, hypotensive anesthesia was given, the operative time is 139 minutes and the total blood loss is $130 \mathrm{~mL}$. Postoperatively, the drain output was 20 $\mathrm{mL}$, and the plasma factor peak level activity was maintained according to the WFH guidelines and nonsteroidal anti-inflammatory (NSAIDs) medication was avoided for the risk of gastrointestinal bleeding. The total hospital stay is 16 days and the patient had symptomatic improvement with a VAS score of 3 and ODI score of 12 after one-year follow-up and without any notable complications. This research study was conducted retrospectively from data obtained for clinical purposes. We consulted extensively with the IRB of Bombay hospital and medical research centre who determined that our study did not need ethical approval. An IRB official waiver of ethical approval was
Table 1. Summary of the patient

\begin{tabular}{lc}
\hline Age & 62 years \\
Procedure & L4-5 MIS-TLIF \\
Operative time & 139 minutes \\
Blood loss & $130 \mathrm{~mL}$ \\
Drain output & $20 \mathrm{~mL}$ \\
Hospital stay & 16 days \\
Pre-op factors (IU/DL) & $60-80$ \\
Post-operative factors (IU/DL) & \\
$1-3$ & $40-60$ \\
$4-6$ & $30-50$ \\
$7-14$ & $20-40$ \\
Blood transfusion & Not done \\
Complications & None \\
\hline
\end{tabular}

MIS-TLIF: minimally invasive transforaminal lumbar interbody fusion.

granted from the IRB of Bombay hospital and medical research centre. Informed consent was obtained from all individual par- 
ticipants included in the study and patients signed informed consent regarding publishing their data and photographs.

\section{Surgical Technique}

Under anesthesia, the patient was prone positioned on a radiolucent operating table. Under fluoroscopic guidance, the level was confirmed and a $3 \mathrm{~cm}$ long paraspinal incision $3-5 \mathrm{~cm}$ away from midline was given on the more symptomatic side for decompression utilizing the same for pedicle screw insertion on that side. Sequential dilatation was done and a tubular retractor with $22 \mathrm{~mm}$ diameter (METRx, Medtronics) was docked over the facet and spino-laminar line. Ipsilateral facetectomy and laminotomy, along with the removal of ligamentum flavum were performed under a microscope to accomplish adequate neural decompression. Utmost care is taken at every step to achieve meticulous hemostasis. Following discectomy and preparation of endplates, an appropriate size interbody cage filled with autologous bone was inserted. The pedicles were cannulated with a cook's needle under fluoroscopic guidance and guidewires were inserted on both sides. The serial dilators were used to dilate over the guidewires and the pedicles were tapped using a cannulated tap. Screws were placed with corresponding screw extenders and the rod was introduced with a device through a proximal stab incision. After placement of locking-cap screws through the screw extenders and application of compression, the screws were torqued and the screw extenders were removed. Closure in layers was performed following wound hemostasis and unlike routine protocol, negative suction drain was kept for one day.

\section{DISCUSSION}

Hemophilia A and B are rare X-chromosome-linked recessive bleeding disorders caused by mutations in the genes causing qualitative and quantitative abnormalities of blood clotting factors VIII and IX, respectively [2]. The prevalence of hemophilia $A$ is 1 in 5,000, and B is 1 in 30,000 male live births [2,3]. The diseased can be grouped as severe, moderate, and mild forms of the diseases, defined by factor plasma levels of $1 \%$ or less, $2 \%$ to $5 \%$, and $6 \%$ to $40 \%$, respectively [3]. They usually present as bleeding after minor trauma or as a spontaneous bleed. Bleeding symptoms often correlate with the degree of residual factor level and the severity of the disease.

It has been described in the descent of Queen Victoria of England and is often called "the disease of the kings" [4]. The earliest description dates back to the second century $\mathrm{AD}$ in the
Babylonian Tribe and the modern history by Dr. John Conrad Otto, where he described an inheritable bleeding disorder. The word "Hemophilia", was first documented by Johann Lukas Schönlein [4].

Surgery in these patients will require additional planning and interaction among the surgeon, anesthetist, and a hematologist because they inevitably result in bleeding, excessive blood loss, and other life-threatening complications. There are some previous studies showing that hemophilia patients can be operated with good results $[5,6]$ following the WFH guidelines. So, optimization of peak plasma factor levels according to the world federation of hemophilia guidelines that is 60-80 IU/DL pre-operatively, 40-60 IU/DL for the first three days post-operatively, 30-50 at four to six post-operative days, 20-40 IU/DL at seven to fourteen post-operative days, is of supreme importance for uneventful surgery [1]. Administration of repeated doses of coagulation factors can lead to the appearance of inhibitors which can cause massive bleeding despite the infusion of coagulation factors. So, checking with the blood inhibitor levels before surgery can save from massive bleeding. Apart from plasma-derived and recombinant coagulation factor concentrates, other agents like desmopressin, tranexamic acid, and epsilon aminocaproic acid also are useful in hemophilia patients [7]. However, they are not used in this case because of the absence of inhibitory factors, normal aPTT values. There is a paradigm shift towards minimally invasive spine surgeries in recent times because of lesser muscle dissection, operative time, blood loss, and other favorable peri-operative factors. These factors can be an added benefit especially in patients with hemophilia to minimize the feared peri-operative complications. The case in our study has total blood loss and operative time similar to previous MISTTLIF studies [8]. This is comparatively low as compared to the case series done by Kobayashi et al. [9] in hemophilia patients. The hospital stay is longer as compared to other studies for intravenous infusion of factors until the 14th postoperative day [8].

Although we had good results in this case report, further studies with a large study group are required. However, this case helps in understanding the protocols of peri-operative management in a hemophilia patient.

\section{CONCLUSION}

Surgeries are safe in hemophilia patients with good planning and a holistic team approach including surgeon, hematologist and anaesthetist with dose adjustment of the coagulation factor to maintain a desirable factor level and having a smooth postoperative recovery. Minimally invasive surgical techniques will 
further help in these patients, as these techniques provide the surgeon with an excellent magnification of the operative field, which enables the use of a smaller incision, less muscle trauma and better hemostasis.

\section{CONFLICT OF INTEREST}

No potential conflict of interest relevant to this article.

\section{REFERENCES}

1. Srivastava A, Santagostino E, Dougall A, Kitchen S, Sutherland M, Pipe SW, et al. WFH Guidelines for the Management of Hemophilia panelists and co-authors. WFH guidelines for the management of hemophilia, 3rd edition. Haemophilia 2020;26 Suppl 6:1-158.

2. Mannucci PM, Tuddenham EG. The hemophilias--from royal genes to gene therapy. N Engl J Med 2001;344:1773-1779.

3. Bolton-Maggs PH, Pasi KJ. Haemophilias A and B. Lancet
2003;361:1801-1809.

4. Schramm W. The history of haemophilia - a short review. Thromb Res 2014;134 Suppl 1:S4-S9.

5. Bastounis E, Pikoulis E, Leppäniemi A, Alexiou D, Tsigris C, Tsetis A. General surgery in haemophiliac patients. Postgrad Med J 2000;76:494-495.

6. Kasper CK, Boylen AL, Ewing NP, Luck JV Jr, Dietrich SL. Hematologic management of hemophilia A for surgery. JAMA 1985;253:1279-1283.

7. Franchini M, Mannucci PM. Past, present and future of hemophilia: a narrative review. Orphanet J Rare Dis 2012;7:24.

8. Hammad A, Wirries A, Ardeshiri A, Nikiforov O, Geiger F. Open versus minimally invasive TLIF: literature review and meta-analysis. J Orthop Surg Res 2019;14:229.

9. Kobayashi K, Imagama S, Ando K, Ito K, Tsushima M, Morozumi M, et al. Perioperative management of patients with hemophilia during spinal surgery. Asian Spine J 2018;12:442445 . 